Baltic Astronomy, vol. 9, 573-577, 2000

\title{
WIDE-FIELD PLATE DATABASE: THE BUCHAREST PLATE CATALOGUES
}

M.K. Tsvetkov ${ }^{1}$, M. Stavinschi ${ }^{2}$, K.P. Tsvetkova ${ }^{1}$, K. Y.Stavrev ${ }^{1}$, G. Bocsa ${ }^{2}$, V. N. Popov ${ }^{1}$ and C. Cristescu ${ }^{2}$

1 Institute of Astronomy, Bulgarian Academy of Sciences, Tsarigradsko Shosse Blvd. 72, Sofia BG-1784, Bulgaria

${ }^{2}$ Astronomical Institute of the Romanian Academy, Str. Cutitul de Argint 5, Bucharest RO-75212, Romania

Received September 10, 2000.

Abstract. We present information on the Bucharest plate catalogues $(0.38 \mathrm{~m}$ refractor and $0.16 \mathrm{~m}$ camera) included in the WideField Plate Database, containing a total of 10533 plates. The primary aims of the observations were to obtain accurate positions of asteroids ( $78 \%$ of all plates) and to serve for different observational campaigns. However, the scientific potential of the archived observations is much larger, with possibilities for investigations of bright visual binaries, long-term variability of the Pleiades red dwarf stars and potentially hazardous near-Earth asteroids.

Key words: astronomical databases - plate archives

\section{INTRODUCTION}

The century-long era of photographic observations in astronomy has now passed over due to the wide application of electronic light detectors more sensitive than photographic emulsion. The heritage of this era, however, remains in numerous plate archives all over the world as a resource for various astronomical investigations, e.g. astrometry, variable objects, phenomena that change on a long timescale. In order to facilitate the use of astronomical plate archives the Wide-Field Plate Database (WFPDB) has been created (Tsvetkov et al. 1998) by merging a large number of plate catalogues from different observatories. The aim of the present paper is to analyze 
574 M. K. Tsvetkov, M. Stavinschi, K. P. Tsvetkova, K. Y. Stavrev et al.

the data in the WFPDB concerning two of the catalogues included in it - the Bucharest plate catalogues - in order to reveal the scientific potential of the archived observations.

\section{ANALYSIS OF THE BUCHAREST PLATE CATALOGUES}

Wide-field photographic observations in the Bucharest Observatory started in 1930 with the $0.38 \mathrm{~m}$ refractor (WFPDB instrument identifier BUC038) and the $0.16 \mathrm{~m}$ camera (BUC016). Computer readable versions of the logbooks and a preliminary analysis of the plate data have been made by Vass et al. (1994) and Vass (1994). Our analysis of the Bucharest plate catalogues is based on data retrieval from the WFPDB and, in contrast to Vass (1994), the two catalogues are examined separately. The WFPDB contains 10353 BUC038 plates and 180 BUC016 plates. Distribution of their centers on the celestial sphere in equatorial coordinates is shown in Fig. 1.

The distributions of the number of plates during the period 19301993 for both plate catalogues are shown in Fig. 2. It is seen that BUC038 was most productive in the 60's and 70's, while BUC016 produced more plates in the 30's.

We find that for BUC038 exposures up to $10 \mathrm{~min}(71 \%)$ are mainly used. For BUC016 the exposures are longer but usually shorter than 1 hour. Multiple exposures are $85 \%$ of all observations for BUC038 and $21 \%$ for BUC016.

The majority of plates in the Bucharest catalogues are devoted to asteroid observations ( $78 \%$ of all plates). This makes the plate collections extremely valuable for the near-Earth asteroid (NEA) investigations - detection, measurements and making accurate predictions for future close encounters. Another aspect of the future use of the archived observations is the photometric investigation of binary stars (more than 80 plates), e.g. observations of close binary eclipsing systems YY CMi and KO Aql obtained in the period 1956-1958 can be found.

The wide-field plates of the Bucharest Observatory archives have been accumulated mainly as a result of the following observational programs: (1) precise photographic positions of minor planets and comets, (2) an inertial system of stellar reference by observing 15 designated asteroids, (3) stellar proper motions, (4) connection between radio (VLBI observations) and optical (FK5) reference frames according to the Kiev program CONFOR, (5) photographic determi- 

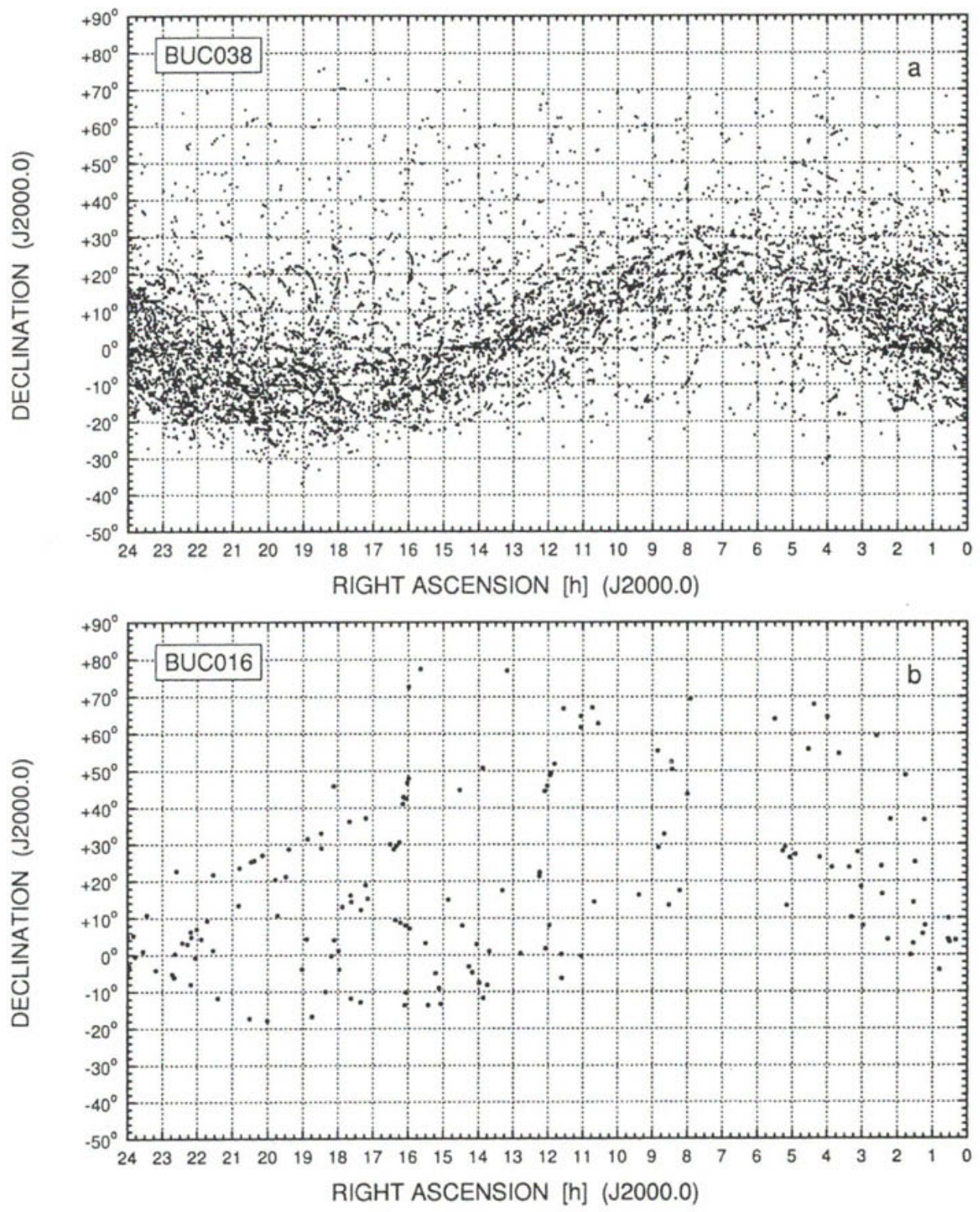

Fig. 1. Distribution on the celestial sphere of the centers of the Bucharest Observatory plates taken with (a) the $0.38 \mathrm{~m}$ refractor (BUC038) and (b) the $0.16 \mathrm{~m}$ camera (BUC016). 
576 M. K. Tsvetkov, M. Stavinschi, K. P. Tsvetkova, K. Y. Stavrev et al.
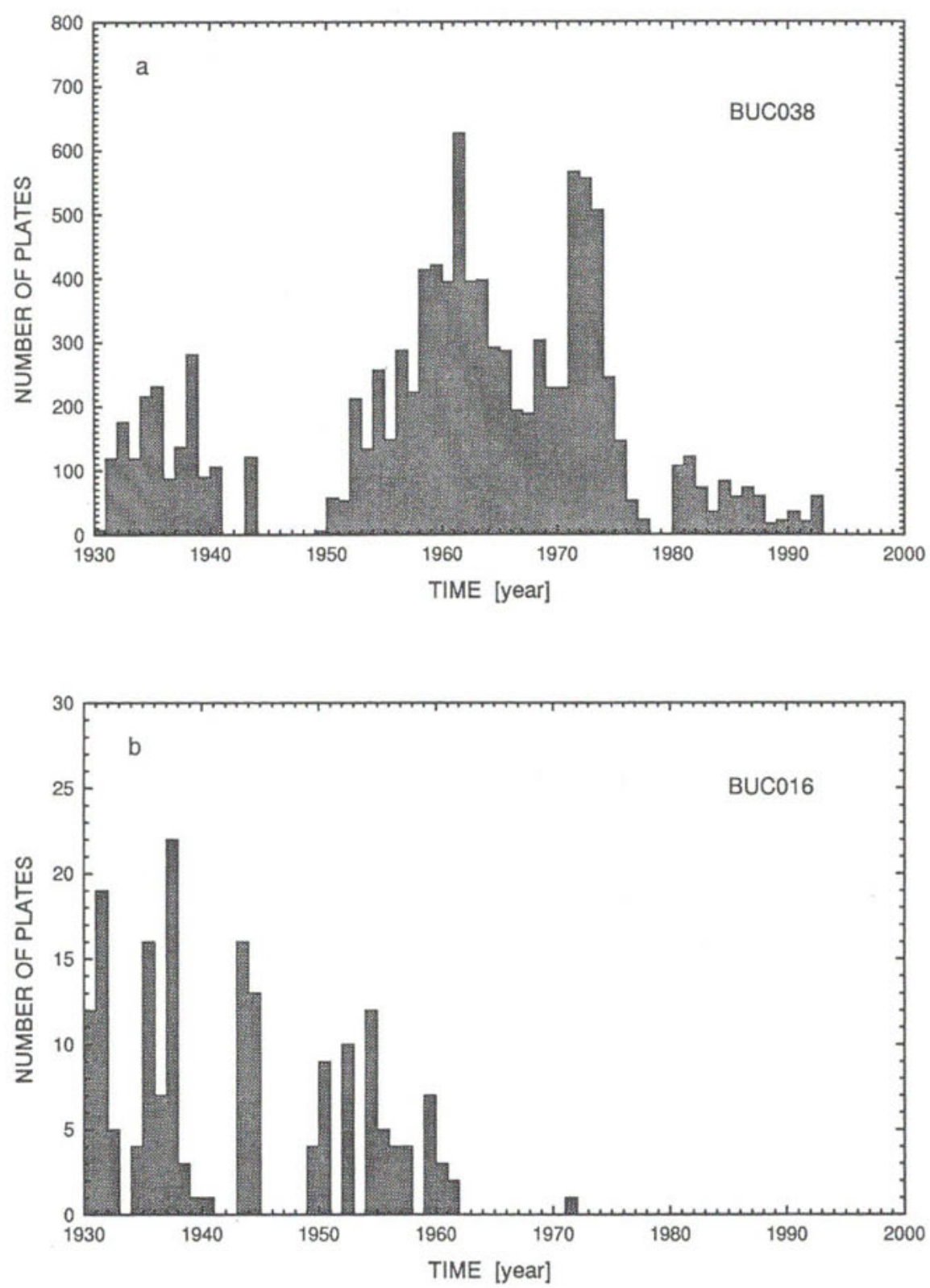

Fig. 2. Number of plates versus time for (a) BUC038 and (b) BUC016. 
nation of the orbits of the Galilean satellites of Jupiter, (6) determination of the brightness of the stellar clusters M13 and the Hyades, (7) photometric studies of closed binaries and Mira Ceti-type variable stars, (8) Moon eclipses, (9) stellar occultations, (10) Venus and Mercury passages, (11) positional measurements of Pluto and Jupiter. The results from these programs are published in the Romanian journals "Studies and Researches in Astronomy and Seismology" (1956-1962), "Studies and Researches in Astronomy" (1963-1974) and "Contributions to Astronomy" (1975), as well as in Astronomische Nachrichten, Journal des Observateurs and the IAU Circular Letters.

\section{CONCLUSIONS}

The future use of the Bucharest archive plates obviously should be connected with the primary aim of the observations - minor planet investigations. However, the scientific potential of the Bucharest plate archive is much larger in comparison with its primary aim. It may be useful for investigation of long-term variability of the Pleiades red dwarf stars, for investigation of bright visual binaries and for the search program of potentially hazardous NEAs.

ACKNOWLEDGMENT. We are grateful to G. Vass for useful discussions.

\section{REFERENCES}

Tsvetkov M. K., Stavrev K.Y., Tsvetkova K.P., Semkov E. H., Mutafov A. S., Michailov M.-E. 1998, in New Horizons from Multi-Wavelength Sky Surveys, eds. B. McLean et al., Kluwer Academic Publishers, Dordrecht, p. 462

Vass G. 1994, Romanian Astron. J., 4, 183

Vass G., Bocsa G., Ionescu V., Alexiu A., Birlan M. 1994, Romanian Astron. J., 4, 179 